\title{
COVID-19 Vaccine Efficacy in Liver Transplant Recipients: A Rising Concern
}

\author{
Mariyam Zahid®, Nuwaira Irshad®, Naveen Azhar®, Jawad Ahmed®
}

Internal Medicine, Dow University of Health Sciences, Karachi, Pakistan

\section{To the Editor}

The severe acute respiratory syndrome coronavirus-2 (SARSCoV-2) has taken millions of lives all around the globe. Presently, the vaccine is the only way to overcome this pandemic and to reduce the further mortality and morbidity related to SARS-CoV-2. Various types of SARS-CoV-2 vaccines have been approved and have demonstrated considerable results in immunocompetent populations. However, the effectiveness of vaccines in solid-organ transplant recipients remains a scientific concern, as they have reduced immunity to vaccines; therefore, they have been excluded from the initial trials. ${ }^{1,2}$

However, two prospective studies assessed the efficacy of $m R N A$ based SARS-CoV-2 vaccine BNT162b2 in liver transplant (LT) recipients compared with young healthcare workers as controls. Rabinowich et al. observed that less than half of the transplant recipients developed antibodies against the SARS-CoV-2 recombinant spike protein (38 of 80 LT recipients [47.5\%]), whereas Rashidi-Alavijeh et al. showed an increased antibody response rate (34 of 43 LT recipients [79\%]). However, both of them were significantly lower than the controls (100\%). The antibody titers were significantly lower in LT recipients with a positive serological response than the control group. This outcome agreed with both studies. Negative serology was significantly associated with the administration of immunosuppressive drugs, particularly high dose steroids and Mycophenolate mofetil, older age, and impaired renal function in the study by Rabinowich et al. ${ }^{3}$ Rashidi-Alavijeh et al. ${ }^{4}$ also indicated Mycophenolate mofetil as a risk factor for lower immunogenicity in LT recipients.

In this crucial time of the COVID-19 pandemic, when the whole world is experiencing the huge loss of human lives, the vaccine is a ray of hope for potentially reducing its transmission and rescuing humankind from the devastating effects of the virus. However, the abovementioned studies have raised serious concerns over the efficacy of the COVID-19 vaccine in LT recipients, as a breakthrough infection, defined as a positive SARS-CoV-2 NAAT after $\geq 14$ days of completing the course of COVID-19 vaccine, could be more worrisome in the LT recipients owing to have decreased protective antibodies and the immunosuppressive state compared to the healthy vaccinated individuals. ${ }^{5}$

Current guidelines suggest that candidates on the list awaiting a solid organ transplantation should be completely vaccinated against the vaccine-preventable diseases, as the efficacy of the vaccines is lower after the transplantation due to the administration of immunosuppressive drugs. ${ }^{6}$ Therefore, the question arises, whether vaccination against COVID-19 before liver transplantation may improve immunogenicity to the vaccines and reduce disease-related mortality and morbidity in LT recipients. This demands further substantial studies in this area. Proper guidelines should be devised regarding the timing of immunization and re-immunization protocols for COVID-19 vaccines in the LT candidates and recipients. In the absence of relevant research, these high-risk individuals should continue following non-pharmacologic interventions (e.g., wearing masks, physical distancing, regular hand washing), even after getting vaccinated. Furthermore, vaccination of family members, healthcare workers, and all those in close contact with these patients should be necessary to reduce the risk of virus transmission. With a prompt attention given to every high-risk group, we could mitigate the adverse consequences of SARS-CoV-2 infection.

Patient Consent for Publication: Not applicable.

Author Contributions: Concept - M.Z.; Writing - M.Z., N.A.; Critical Review - N.I., N.A., J.A.

Conflict of Interest: The authors have no conflict of interest to declare.

\section{REFERENCES}

1. Blasi F, Gramegna A, Sotgiu G, et al. SARS-CoV-2 vaccines: A critical perspective through efficacy data and barriers to herd immunity. Respir Med. 2021;180:106355. [CrossRef]

2. Kim YJ, Kim SI. Vaccination strategies in patients with solid organ transplant: evidences and future perspectives. Clin Exp Vaccine Res. 2016;5:125-131. [CrossRef]

3. Rabinowich L, Grupper A, Baruch R, et al. Low immunogenicity to SARS-CoV-2 vaccination among liver transplant recipients. J Hepatol. 2021;75:435-438. [CrossRef]

Corresponding author: Mariyam Zahid, Internal Medicine, Dow University of Health Sciences, Karachi, Pakistan

e-mail: zahidmariyam2@gmail.com

Received: September 2, 2021 Accepted: December 1, 2021 Available Online Date: January 18, 2022 • DOI: 10.4274/balkanmedj.galenos.2021.2021-9-7

Available at www.balkanmedicaljournal.org

ORCID iDs of the authors: M.Z. 0000-0002-3015-0347; N.I. 0000-0003-1493-2979; N.A. 0000-0003-3596-4945; J.A. 0000-0001-5651-1916.

Cite this article as:

Zahid M, Irshad N, Azhar N, Ahmed J. COVID-19 Vaccine Efficacy in Liver Transplant Recipients: A Rising Concern. Balkan Med J.; 2022; 39(1):78-9.

Copyright@Author(s) - Available online at http://balkanmedicaljournal.org/ 
4. Rashidi-Alavijeh J, Frey A, Passenberg M, et al. Humoral Response to SARS-Cov-2 Vaccination in Liver Transplant Recipients-A Single-Center Experience. Vaccines (Basel). 2021;9:738. [CrossRef]

5. Malinis M, Cohen E, Azar MM. Effectiveness of SARS-CoV-2 vaccination in fully vaccinated solid organ transplant recipients. Am J Transplant. 2021;21:2916-2918. [CrossRef]
6. Stucchi RSB, Lopes MH, Kumar D, Manuel O. Vaccine Recommendations for SolidOrgan Transplant Recipients and Donors. Transplantation. 2018;102(suppl 2):72-80. [CrossRef] 